\title{
Fourier's Law for a Harmonic Crystal with Self-Consistent Stochastic Reservoirs ${ }^{1}$
}

\author{
Federico Bonetto, ${ }^{2}$ Joel L. Lebowitz, ${ }^{3}$ and Jani Lukkarinen ${ }^{4}$
}

Received July 17, 2003; accepted January 8, 2004

We consider a $d$-dimensional harmonic crystal in contact with a stochastic Langevin type heat bath at each site. The temperatures of the "exterior" left and right heat baths are at specified values $T_{L}$ and $T_{R}$, respectively, while the temperatures of the "interior" baths are chosen self-consistently so that there is no average flux of energy between them and the system in the steady state. We prove that this requirement uniquely fixes the temperatures and the self consistent system has a unique steady state. For the infinite system this state is one of local thermal equilibrium. The corresponding heat current satisfies Fourier's law with a finite positive thermal conductivity which can also be computed using the Green-Kubo formula. For the harmonic chain $(d=1)$ the conductivity agrees with the expression obtained by Bolsterli, Rich, and Visscher in 1970 who first studied this model. In the other limit, $d \gg 1$, the stationary infinite volume heat conductivity behaves as $\left(\ell_{d} d\right)^{-1}$ where $\ell_{d}$ is the coupling to the intermediate reservoirs. We also analyze the effect of having a non-uniform distribution of the heat bath couplings. These results are proven rigorously by controlling the behavior of the correlations in the thermodynamic limit.

KEY WORDS: Fourier's law; harmonic crystal; non-equilibrium systems; thermodynamic limit; Green-Kubo formula.

\section{INTRODUCTION}

Our understanding of non-equilibrium systems is at the present time very incomplete. In particular, we still have no model Hamiltonian system for which Fourier's law has been proven rigorously. A review of the problems

\footnotetext{
${ }^{1}$ Dedicated to Elliott Lieb on the occasion of his 70 th birthday.

${ }^{2}$ School of Mathematics, Georgia Institute of Technology, Atlanta, Georgia 30332.

${ }^{3}$ Department of Mathematics, Rutgers University, Piscataway, New Jersey 08854.

${ }^{4}$ Centre for Mathematical Sciences, Munich University of Technology, 85747 Garching, Germany; e-mail: jlukkari@ma.tum.de
} 
and of the few known exact results related to Fourier's law is given in refs. 1 and 2 which also contains a survey of recent numerical results.

Here we study, in a mathematically rigorous manner, the microscopic structure of the stationary non-equilibrium state of a "self-consistent harmonic crystal," a model introduced by Bolsterli, Rich, and Visscher (BRV) in refs. 3 and 4. This is a $d$-dimensional system of $N_{1} \times \cdots \times N_{d}$ oscillators whose time-evolution is given by a combination of Hamiltonian and stochastic dynamics. The Hamiltonian is composed of harmonic nearest neighbor and "on-site" potentials and the stochastic part comes from coupling each particle in the chain to its own heat bath.

We want to describe a situation where we have a temperature gradient in one direction (the "first" with $N_{1}$ oscillators) while the temperature is uniform in the remaining $d-1$ directions, on which we impose periodic boundary conditions. The temperatures of the heat baths of the end-point particles in the first direction are fixed to given values $T_{L}$ and $T_{R}$, while the temperatures of the interior heat baths are chosen self-consistently by the requirement that there is no energy flux, on average, between any such reservoir and the system in the steady state. From a physical point of view, we may think of the interior heat reservoirs as representing schematically the effect of degrees of freedom not included in the Hamiltonian.

Using numerical studies and non-rigorous arguments, BRV found that in the case $d=1$ (chain) the (kinetic) temperature profile of the system in its steady state is linear, with a heat flux proportional to $N^{-1}$ for large $N$. This corresponds to the self-consistent system having a finite, temperature independent, thermal conductivity. ${ }^{(1,2)}$ These results are in sharp contrast to those found earlier by Rieder, Lebowitz, and Lieb, ${ }^{(5)}$ who studied a system with the same Hamiltonian dynamics, but with heat baths acting only at the boundaries. They found that the system had an infinite conductivity and a constant temperature profile away from the ends, results later generalized to the higher dimensional case by Nakazawa. ${ }^{(6)}$

In this paper, we provide a rigorous proof that the steady state of the self-consistent system has indeed the properties found by BRV for the $d=1$ case in refs. 3 and 4 , and we extend the results to cover all $d \geqslant 1$. More precisely, we show that, in the limit where all $N_{i} \rightarrow \infty$, the steady state is a local equilibrium state ${ }^{(7)}$ with a temperature profile satisfying Fourier's law with a finite, temperature independent, thermal conductivity.

We deal first with the $d=1$ case, and consider the higher dimensional case only in Section 7. We define the model and solve its dynamics with a given temperature profile in Section 2. We then turn to the self-consistency condition in Section 3, proving in particular, that the self-consistent profile is always uniquely determined by the boundary temperatures. Section 4 contains our main results: we prove there the local equilibrium property 
and Fourier's law. In Section 5, we use the explicit solution to show that the Green-Kubo formula holds for this system. In Section 6, we briefly analyze the case where the couplings to the heat baths are non-uniform, and we conclude that the local macroscopic heat conductivity is proportional to the inverse of the local average of the couplings.

Finally in Section 7, we first show how one can map the higher dimensional self-consistent system with periodic boundary condition on all directions but the first into a set of one-dimensional chains, and then apply the earlier results to derive a generalization to the higher dimensional case. We show there that for large $d$ the conductivity behaves as $\left(\ell_{d} d\right)^{-1}$ where $\ell_{d}$ is the coupling to the intermediate reservoirs. Some technical details of the calculations are collected in the appendices.

\section{DYNAMICS AND THE STATIONARY STATE}

To start with, we consider a chain of $N$ oscillators with a Hamiltonian

$$
H(\mathbf{q}, \mathbf{p})=\sum_{i=1}^{N}\left[\frac{1}{2} p_{i}^{2}+u\left(q_{i}\right)\right]+\sum_{i=1}^{N+1} v\left(q_{i}-q_{i-1}\right),
$$

where $\mathbf{q}$ and $\mathbf{p}$ are vectors in $\mathbb{R}^{N}$, we set $q_{0}=0=q_{N+1}$, and we have

$$
u(q)=\frac{1}{2} \gamma^{2} q^{2} \quad \text { and } \quad v(q)=\frac{1}{2} \omega^{2} q^{2}
$$

with $\gamma, \omega>0$. In addition, the oscillator at each site $i$ is coupled to a Langevin heat bath at temperature $T_{i} \geqslant 0$, with a coupling strength $\lambda>0$. As in ref. 5, the time-evolution of the system is then given by the stochastic differential equations,

$$
\dot{\mathbf{X}}=-A \mathbf{X}+\Sigma \dot{\mathbf{W}}
$$

where $\mathbf{X}=(\mathbf{q}, \mathbf{p})$ is the phase-space vector, the $\dot{W}_{i}$ are independent white noises and $A$ and $\Sigma$ are $2 N$ by $2 N$ matrices given by

$$
A=\left(\begin{array}{cc}
0 & -I \\
\Phi & \Lambda
\end{array}\right) \quad \text { and } \quad \Sigma=\left(\begin{array}{cc}
0 & 0 \\
0 & \sqrt{2 \Lambda \mathscr{T}}
\end{array}\right)
$$

Here $I$ is the unit $N$ by $N$ matrix, $\Lambda_{i j}=\delta_{i j} \lambda, \mathscr{T}_{i j}=\delta_{i j} T_{i}$, and

$$
\Phi=\omega^{2}\left(-\Delta+v^{2} I\right)
$$


where $v^{2}=\gamma^{2} / \omega^{2}$ and $\Delta$ denotes the discrete Laplacian with Dirichlet boundary conditions:

$$
\Delta_{i j}=-2 \delta_{i, j}+\delta_{i-1, j}+\delta_{i+1, j} .
$$

Equations (2.3) define an Ornstein-Uhlenbeck process whose solution with initial data $\mathbf{X}(0)$ is given by the stochastic integral (for details, see, e.g., Chap. 5 in ref. 8)

$$
\mathbf{X}(t)=\mathrm{e}^{-t A} \mathbf{X}(0)+\int_{0}^{t} \mathrm{~d} s \mathrm{e}^{-(t-s) A} \Sigma \dot{\mathbf{W}}(s) .
$$

This is a Gaussian process, determined uniquely by its mean and covariance which can be computed directly from (2.6). However, for latter use we assume now that $\mathbf{X}(0)$ is distributed according to a Gaussian measure with a mean $\mathbf{X}_{0}$ and a covariance $C_{0}$ - the deterministic case is then obtained by setting $C_{0}=0$. Then the mean evolves by

$$
\langle\mathbf{X}(t)\rangle=\mathrm{e}^{-t A} \mathbf{X}_{0},
$$

and for the covariance $C\left(t^{\prime}, t\right) \equiv\left\langle\left[\mathbf{X}\left(t^{\prime}\right)-\left\langle\mathbf{X}\left(t^{\prime}\right)\right\rangle\right] \otimes[\mathbf{X}(t)-\langle\mathbf{X}(t)\rangle]\right\rangle$ we get

$$
C\left(t^{\prime}, t\right)= \begin{cases}\mathrm{e}^{-\left(t^{\prime}-t\right) A} C(t, t) & \text { if } \quad t^{\prime} \geqslant t \\ C\left(t^{\prime}, t^{\prime}\right) \mathrm{e}^{-\left(t-t^{\prime}\right) A^{T}} & \text { if } t^{\prime} \leqslant t\end{cases}
$$

with

$$
C(t, t)=\mathrm{e}^{-t A} C_{0} \mathrm{e}^{-t A^{T}}+\int_{0}^{t} \mathrm{~d} s \mathrm{e}^{-s A} \Sigma^{2} \mathrm{e}^{-s A^{T}} .
$$

We show in Appendix A that for any $\alpha$ satisfying

$$
0<\alpha<\min \left\{\frac{\lambda}{2}, \frac{\gamma^{2}}{\lambda}\right\}
$$

we can find a constant $c<\infty$ such that for all $t>0$ and for all $N$

$$
\left\|\mathrm{e}^{-t A}\right\| \leqslant c \mathrm{e}^{-t \alpha} \text {. }
$$

The uniform exponential decay of $\mathrm{e}^{-t A}$ implies that there is an exponentially fast convergence in the microscopic scale to a unique stationary state, which is Gaussian with mean $\mathbf{0}$ and covariance $S$,

$$
S=\int_{0}^{\infty} \mathrm{d} s \mathrm{e}^{-s A} \Sigma^{2} \mathrm{e}^{-s A^{T}} .
$$


This $S$ is the unique solution of the equation

$$
A S+S A^{T}=\Sigma^{2},
$$

which we solve following ref. 3 . We divide $S$ into $N$ by $N$ components,

$$
S=\left(\begin{array}{cc}
U & Z \\
Z^{T} & V
\end{array}\right)
$$

and get the following four equations equivalent to (2.12):

$$
\begin{aligned}
Z=-Z^{T}, \quad V & =\frac{1}{2}(\Phi U+U \Phi)+\frac{1}{2}(Z \Lambda-\Lambda Z), \\
Z \Lambda+\Lambda Z & =\Phi U-U \Phi, \\
\Lambda(\mathscr{T}-V)+(\mathscr{T}-V) \Lambda & =\Phi Z-Z \Phi .
\end{aligned}
$$

A diagonalization of the discrete Laplacian yields

$$
\Phi=F Y F^{T},
$$

where $Y_{k l}=\mu_{k} \delta_{k l}, \mu_{k}$ are the eigenvalues of $\Phi$ :

$$
\frac{\mu_{k}}{\omega^{2}}=v^{2}+4 \sin ^{2}\left(\frac{\pi k}{2(N+1)}\right),
$$

and $F$ is the orthonormal matrix

$$
F_{k l}=\sqrt{\frac{2}{N+1}} \sin \left(\frac{\pi k l}{N+1}\right) .
$$

As shown in Appendix B, any block $B$ of the covariance matrix (i.e., $B=U, V$, or $Z$ ) can then be obtained by a linear transformation of the form

$$
B_{i j}=\sum_{r=1}^{N} B_{i j}^{(r)} T_{r}
$$

where

$$
B_{i j}^{(r)}=\sum_{k, l=1}^{N} F_{i k} F_{j l} f^{(B)}\left(c_{k}, c_{l}\right) F_{r k} F_{r l}
$$

and

$$
c_{k}=\cos \left(\frac{\pi k}{N+1}\right) .
$$


The functions $f^{(B)}$ for the different choices of $B$ are given by

$$
\begin{aligned}
& f^{(U)}(x, y)=\frac{\lambda^{2}}{\omega^{4}} \frac{1}{G(x, y)} \\
& f^{(V)}(x, y)=1-\frac{(x-y)^{2}}{G(x, y)} \\
& f^{(z)}(x, y)=\frac{\lambda}{\omega^{2}} \frac{y-x}{G(x, y)}
\end{aligned}
$$

where

$$
G(x, y)=(x-y)^{2}+\frac{\lambda^{2}}{\omega^{2}}\left(v^{2}+2-x-y\right) .
$$

When $T_{i}=T$ for all $i$, only the values with $k=l$ contribute in Eq. (2.17). The stationary covariance is then given by

$$
S^{(\mathrm{eq}, T)}=T\left(\begin{array}{cc}
\Phi^{-1} & 0 \\
0 & I
\end{array}\right)
$$

and the stationary measure is the Gibbs measure at temperature $T$.

\subsection{Energy Current}

We define the local energy of particle $i$ by

$$
H_{i}(\mathbf{q}, \mathbf{p})=\frac{1}{2} p_{i}^{2}+u\left(q_{i}\right)+\frac{1}{2}\left[v\left(q_{i}-q_{i-1}\right)+v\left(q_{i+1}-q_{i}\right)\right]
$$

for $i=2, \ldots, N-1$. The boundary terms $(i=1, N)$ are defined similarly, but with double the interaction energy contribution from the connections to $q_{0}=0$ and to $q_{N+1}=0$. With these definitions,

$$
H(\mathbf{q}, \mathbf{p})=\sum_{i=1}^{N} H_{i}(\mathbf{q}, \mathbf{p})
$$

and

$$
\frac{\mathrm{d}}{\mathrm{d} t}\left\langle H_{i}(t)\right\rangle=-\left[\left\langle J_{i}(t)\right\rangle-\left\langle J_{i-1}(t)\right\rangle\right]+\left\langle R_{i}(t)\right\rangle,
$$

where

$$
J_{i}(\mathbf{q}, \mathbf{p})=-\frac{\omega^{2}}{2}\left(q_{i+1}-q_{i}\right)\left(p_{i}+p_{i+1}\right), \quad i=1, \ldots, N-1,
$$


$J_{i}=0$ for $i=0, N$, and

$$
R_{i}(\mathbf{q}, \mathbf{p})=\lambda\left(T_{i}-p_{i}^{2}\right)
$$

The $J_{i}$ correspond to the energy currents inside the system while $R_{i}$ gives the energy flux from the $i$ th reservoir to the $i$ th oscillator.

The corresponding expectation values in the stationary state are

$$
\left\langle J_{i}\right\rangle_{S}=\frac{\omega^{2}}{2}\left(-Z_{i+1, i}-Z_{i+1, i+1}+Z_{i, i}+Z_{i, i+1}\right)=\omega^{2} Z_{i, i+1},
$$

where we have used the antisymmetry of $Z$, and

$$
\left\langle R_{i}\right\rangle_{S}=\lambda\left(T_{i}-V_{i i}\right)
$$

\section{SELF-CONSISTENCY CONDITION}

As described in the introduction, we let the end-point temperatures $T_{1}=T_{L}$ and $T_{N}=T_{R}$ be given independently of $N$. Then we want to choose $T_{i}$ for $i=2, \ldots, N-1$ in such a way that $\left\langle R_{i}\right\rangle_{S}=0$ or, by (2.25), so that

$$
T_{i}=V_{i i}
$$

By (2.11), the kinetic temperature vector $\left(V_{i i}\right)_{i=1}^{N}$ depends linearly on the imposed temperature vector $\mathbf{T}$. Therefore, all solutions to the selfconsistency condition can be obtained using the equation

$$
T_{i}=T_{R}+\left(T_{L}-T_{R}\right) T_{i}^{(1,0)}
$$

where $\mathbf{T}^{(1,0)}$ is a solution of the problem with $T_{L}=1$ and $T_{R}=0$. The set of all such $\mathbf{T}^{(1,0)}$ form a convex set, i.e., any non-uniqueness in the solution of the self-consistency condition would imply the existence of a whole continuum of solutions. We shall now prove that for our model the solution to the self-consistency problem is unique.

Let us denote the above linear mapping from imposed to kinetic temperatures by $M$, i.e., $V_{i i}=\sum_{j} M_{i j} T_{j}$. It follows straightforwardly from (2.11) that $M_{i j} \geqslant 0$ for all pairs $i, j$, and by the explicit solution given in (2.16)-(2.20) we have

$$
M_{i j}=V_{i i}^{(j)}=\sum_{k, l=1}^{N} F_{i k} F_{i l} f_{k l} F_{j k} F_{j l},
$$


where

$$
f_{k l}=f^{(V)}\left(c_{k}, c_{l}\right)=1-\frac{\left(c_{k}-c_{l}\right)^{2}}{G\left(c_{k}, c_{l}\right)} \geqslant 0 .
$$

By (3.3), $M$ is then symmetric and satisfies for all $i$

$$
\sum_{j=1}^{N} M_{i j}=\sum_{j=1}^{N} M_{j i}=1,
$$

which imply that $M$ is, in fact, a doubly stochastic matrix.

Theorem 3.1. For any given end-point temperatures $T_{L}, T_{R} \geqslant 0$, there is a unique, positive temperature profile which satisfies the self-consistency condition. In addition, all temperatures in the profile lie between the end-point temperatures.

Proof. Let $\mathbf{T}$ be a self-consistent profile and define

$$
a_{i}= \begin{cases}T_{i}-T_{R}, & \text { for } i=2, \ldots, N-1 \\ 0, & \text { if } i=1 \text { or } i=N\end{cases}
$$

Clearly a belongs to the subspace

$$
W=\left\{\mathbf{x} \in \mathbb{R}^{N} \mid x_{1}=0=x_{N}\right\} .
$$

Let us denote the orthogonal projection to the subspace $W$ by $P_{W}$, and define $Q=P_{W} M P_{W}$. Using (3.4) in (3.1) we get the equation

$$
\mathbf{a}=Q \mathbf{a}+\mathbf{b}
$$

where the vector $\mathbf{b}$ is defined by

$$
b_{i}= \begin{cases}M_{i 1}\left(T_{L}-T_{R}\right), & \text { for } i=2, \ldots, N-1, \\ 0, & \text { if } i=1 \text { or } i=N .\end{cases}
$$

We shall later prove in Corollary 3.3 that $\|Q\|<1$. Then for any $\mathbf{b} \in \mathbb{R}^{N}$, Eq. (3.5) has a unique solution given by

$$
\mathbf{a}=\sum_{n=0}^{\infty} Q^{n} \mathbf{b},
$$


and the self-consistent profile must thus satisfy

$$
T_{i}=T_{R}+\sum_{n=0}^{\infty}\left(Q^{n} \mathbf{b}\right)_{i}, \quad \text { for } \quad i=2, \ldots, N-1 .
$$

As the profile defined by (3.6) also satisfies (3.1), this proves the existence and uniqueness of the self-consistent profile. Repeating the above computation for $\mathbf{a}^{\prime}=P_{W}\left(\mathbf{T}-T_{L} \mathbf{1}\right)$ instead of $\mathbf{a}$, and then using the positivity of $M_{i j}$ in the equations corresponding to (3.6), proves that $T_{R} \leqslant T_{i} \leqslant T_{L}$ for all $i$ when $T_{R} \leqslant T_{L}$, and that $T_{L} \leqslant T_{i} \leqslant T_{R}$ when $T_{R} \geqslant T_{L}$.

To conclude the proof, we still need to prove that $\|Q\|<1$. This will follow from the following lemma:

Lemma 3.2. Let $c$ be a constant which satisfies

$$
G(x, y) \leqslant \frac{c}{2}
$$

for all $x, y \in[-1,1]$. Then, for any vector $\mathbf{x} \in \mathbb{R}^{N}$,

$$
\mathbf{x}^{T}(I-M) \mathbf{x} \geqslant \frac{1}{c}\|D \mathbf{x}\|^{2}
$$

where $D$ is the "finite difference operator" defined by

$$
(D \mathbf{x})_{i}= \begin{cases}x_{i}-x_{i+1}, & \text { for } 1 \leqslant i \leqslant N-1 \\ 0, & \text { for } i=N .\end{cases}
$$

Proof. Let $\mathbf{x} \in \mathbb{R}^{N}$, and define $\tilde{x}_{k l}=\sum_{i=1}^{N} F_{i k} F_{i l} x_{i}$ for all $k, l$. Then by (3.3) and the assumption $G\left(c_{k}, c_{l}\right) \leqslant c / 2$,

$$
\begin{aligned}
\mathbf{x}^{T}(I-M) \mathbf{x} & \geqslant \frac{2}{c} \sum_{k, l=1}^{N} \tilde{x}_{k l}^{2}\left(c_{k}-c_{l}\right)^{2} \\
& =\frac{2}{c} \sum_{i, j=1}^{N} x_{i} x_{j} \sum_{k, l=1}^{N} F_{i k} F_{i l} F_{j k} F_{j l}\left(\left(1-c_{k}\right)-\left(1-c_{l}\right)\right)^{2} .
\end{aligned}
$$

But by an explicit computation, we have for all $i, j$,

$$
2 \sum_{k, l=1}^{N} F_{i k} F_{i l} F_{j k} F_{j l}\left(\left(1-c_{k}\right)-\left(1-c_{l}\right)\right)^{2}=\left(\Delta^{2}\right)_{i j} \delta_{i j}-\left(\Delta_{i j}\right)^{2}=\left(D^{T} D\right)_{i j},
$$

and the inequality (3.7) has been derived. 
For the applications, we note that a constant $c$ satisfying the requirement of the lemma can always be found.

Corollary 3.3. $\|Q\|<1$.

Proof. Since $W$ is finite-dimensional and $Q=P_{W} Q P_{W}$ is symmetric, it will be enough to show that for all $\mathbf{x} \in W$ with $\|\mathbf{x}\| \leqslant 1$

$$
0 \leqslant \mathbf{x}^{T} Q \mathbf{x}<1 .
$$

But for such $\mathbf{x}, \mathbf{x}^{T} Q \mathbf{x}=\mathbf{x}^{T} M \mathbf{x}$, and the lemma together with $f_{k l} \geqslant 0$ yields

$$
0 \leqslant \mathbf{x}^{T} M \mathbf{x} \leqslant 1-\mathbf{x}^{T}(I-M) \mathbf{x} \leqslant 1-\frac{1}{c}\|D \mathbf{x}\|^{2} .
$$

If $D \mathbf{x}=\mathbf{0}$, then $x_{i}=x_{1}=0$ for all $i$, and we must now have either $\|D \mathbf{x}\|>0$, when (3.9) implies (3.8), or $\mathbf{x}=0$, when (3.8) is trivially true.

\section{FOURIER'S LAW}

In this section we first derive a number of technical estimates which will be necessary to control the behavior of the system in the thermodynamic limit. We then show that the self-consistent steady state is microscopically a local equilibrium state with a heat flux satisfying Fourier's law.

\subsection{Exponential Decay of Correlations}

Let $f$ denote any one of the three functions $f^{(B)}$ defined by Eq. (2.19). Clearly, $f$ is a rational function, analytic everywhere but at the zeroes of $G$ in (2.20). On the other hand,

$$
G(x, y) \geqslant \frac{\lambda^{2} v^{2}}{\omega^{2}}=\lambda^{2} \frac{\gamma^{2}}{\omega^{4}}
$$

for all $x, y \in[-1,1]$, and since we have assumed that $\gamma>0$, there are no zeroes of $G$ inside $[-1,1]^{2}$.

This implies that the function $f(\cos \cdot, \cos \cdot)$, which enters in $(2.17)$, is analytic in some neighborhood region of $[-\pi, \pi]^{2}$ in $\mathbb{C}^{2}$. In particular, its Fourier series converges pointwise, and we have for all $x, y \in \mathbb{R}$,

$$
f(\cos x, \cos y)=\sum_{m, n=-\infty}^{\infty} \cos (m x) \cos (n y) \hat{f}(m, n) .
$$


Applying this with $f=f^{(B)}$ in (2.17) we get after some straightforward algebra,

$$
B_{i j}^{(r)}=\hat{f}_{N}(i-r, j-r)+\hat{f}_{N}(i+r, j+r)-\hat{f}_{N}(i-r, j+r)-\hat{f}_{N}(i+r, j-r)
$$

where $\hat{f}_{N}$ is defined by

$$
\hat{f}_{N}(m, n)=\sum_{k, l \in \mathbb{Z}} \hat{f}(m+2(N+1) k, n+2(N+1) l) .
$$

By the above mentioned analyticity, the Fourier coefficients $\hat{f}$ decay exponentially. From this the following behavior for $\hat{f}_{N}$ is easily derived:

Lemma 4.1. For any block $B$, define $\hat{f}_{N}$ by (4.4) using $f=f^{(B)}$. Then there are strictly positive, $N$-independent constants $a$ and $\alpha$ such that, for any $m, n \in \mathbb{Z}$,

$$
\left|\hat{f}_{N}(m, n)\right| \leqslant a \mathrm{e}^{-\alpha\left(\left|m^{\prime}\right|+\left|n^{\prime}\right|\right)}
$$

where $m^{\prime}=(m \bmod 2(N+1)) \in\{-N, \ldots, N+1\}$ and $n$ defines $n^{\prime}$ similarly.

\subsection{Local Equilibrium}

Consider some temperature profile $\mathbf{T}=\left(T_{i}\right)_{i=1}^{N}$, and let its maximum nearest neighbor variation be denoted by $\varepsilon_{N}$, i.e., with $D$ defined as in Lemma 3.2, let

$$
\varepsilon_{N}=\max _{i}\left|(D \mathbf{T})_{i}\right|=\max _{i<N}\left|T_{i}-T_{i+1}\right|
$$

We shall prove in this section that, if $\varepsilon_{N} \ll 1$ and $\max _{i} T_{i}$ is bounded, the local microscopic properties of the stationary measure near a site $i$ can be well approximated by using the equilibrium measure with the temperature $T_{i}$. This will, in particular, justify our identification of the parameter $T_{i}$ as a local temperature.

The main ingredient of the proof is the following corollary to Lemma 4.1:

Corollary 4.2. For any block $B$, there is an $N$-independent constant $a^{\prime}$, such that for all $i, j \in\{1, \ldots, N\}$,

$$
\sum_{r=1}^{N}|i-r|\left|B_{i j}^{(r)}\right| \leqslant a^{\prime}
$$


Proof. Apply Eq. (4.3), the triangle inequality, and Lemma 4.1 to replace $\left|B_{i j}^{(r)}\right|$ in (4.7) by four exponential terms. The estimate follows straightforwardly.

Let $B$ be any block of the stationary covariance matrix, and let $i$, $j \in\{1, \ldots, N\}$. As it takes $|r-i|$ "steps" to get from a site $r$ to the site $i$, we have the obvious bound

$$
\left|T_{r}-T_{i}\right| \leqslant|r-i| \varepsilon_{N}
$$

Then Corollary 4.2 immediately yields the estimate

$$
\left|B_{i j}-T_{i} \sum_{r=1}^{N} B_{i j}^{(r)}\right| \leqslant a^{\prime} \varepsilon_{N} .
$$

The value of $T_{i} \sum_{r=1}^{N} B_{i j}^{(r)}$ gives the component of the stationary covariance matrix when all the temperatures are set equal to $T_{i}$, i.e., the equilibrium covariance $B^{\left(\mathrm{eq}, T_{i}\right)}$ at temperature $T_{i}$, see (2.21). As $a^{\prime}$ in (4.9) can be chosen independently of $N$, we have now shown that all paircorrelations satisfy

$$
B_{i j}=B_{i j}^{\left(\mathrm{eq}, T_{i}\right)}+O\left(\varepsilon_{N}\right) .
$$

As both the equilibrium measure and the above stationary measure are Gaussian, we can also conclude that all finite correlations can be approximated by the local equilibrium values, with an error which vanishes when $\varepsilon_{N} \rightarrow 0$.

\subsection{Self-Consistent Current and Fourier's Law}

Let $\mathbf{T}$ now denote the self-consistent profile which by Theorem 3.1 is unique and is bounded by $T_{L}$ and $T_{R}$. Then by (2.13) and (2.24) we get for all $i=2, \ldots, N-1$,

$$
0=\lambda(\mathscr{T}-V)_{i i}=\frac{1}{2}(\Phi Z-Z \Phi)_{i i}=\omega^{2}\left(-Z_{i-1, i}-Z_{i+1, i}\right)=\left\langle J_{i}\right\rangle_{S}-\left\langle J_{i-1}\right\rangle_{S} .
$$

Therefore, the steady state current is constant throughout the chain:

$$
\left\langle J_{i}\right\rangle_{S}=J^{(N)}, \quad \text { for } \quad i=1, \ldots, N-1 .
$$

The same equations also imply the following relation between the local steady state current and the $q-q$ correlations: for all $i=1, \ldots, N-1$,

$$
\begin{aligned}
\left\langle J_{i}\right\rangle_{S} & =\omega^{2} Z_{i, i+1}=\frac{\omega^{2}}{2 \lambda}(\Phi U-U \Phi)_{i, i+1} \\
& =\frac{\omega^{4}}{2 \lambda}\left(U_{i i}+U_{i, i+2}-U_{i-1, i+1}-U_{i+1, i+1}\right),
\end{aligned}
$$


where we define $U_{i j}=0$ when $i$ or $j \notin\{1, \ldots, N\}$. Summing (4.12) over all the indices $i$, we get by using Eq. (4.11)

$$
(N-1) J^{(N)}=\frac{\omega^{4}}{2 \lambda}\left(U_{11}-U_{N N}\right) .
$$

Let then $D$ and $c$ be given as in Lemma 3.2, and as before let $\varepsilon_{N}=\max _{i}\left|(D \mathbf{T})_{i}\right|$. Then the magnitude of the current and $\varepsilon_{N}$ are related by the formula

$$
\varepsilon_{N} \leqslant \sqrt{\frac{c}{\lambda}\left(T_{L}-T_{R}\right) J^{(N)}} .
$$

To see this, first let $\mathbf{x}=(I-M) \mathbf{T}$ where the matrix $M$ was defined in Section 3. Since $\mathbf{T}$ is self-consistent, $x_{i}=0$ except possibly at the endpoints. But then by (3.4), $x_{1}+x_{N}=\sum_{i} x_{i}=0$, and thus also $x_{N}=-x_{1}$. Therefore, (4.14) follows from Lemma 3.2, since then

$$
x_{1}\left(T_{L}-T_{R}\right)=\mathbf{T}^{T}(I-M) \mathbf{T} \geqslant \frac{1}{c}\|D \mathbf{T}\|^{2} \geqslant \frac{1}{c} \varepsilon_{N}^{2},
$$

and, by (2.13), $x_{1}=T_{1}-V_{11}=\frac{1}{2 \lambda}(\Phi Z-Z \Phi)_{11}=\left\langle J_{1}\right\rangle / \lambda$.

Using (4.14) we can estimate the error made when the terms on the right side of (4.13) are replaced by their equilibrium values. Equation (4.9) and the explicit form of the equilibrium covariance given in (2.21) yield

$$
\left|(N-1) J^{(N)}-\frac{\omega^{4}}{2 \lambda}\left(\Phi_{11}^{-1} T_{L}-\Phi_{N N}^{-1} T_{R}\right)\right| \leqslant a^{\prime} \frac{\omega^{2}}{\lambda} \sqrt{\frac{c}{\lambda}\left(T_{L}-T_{R}\right) J^{(N)}}
$$

where $a^{\prime}$ is chosen as in Corollary 4.2 for the block $B=U$.

It follows from symmetry that $\Phi_{N N}^{-1}=\Phi_{11}^{-1}$, which has the limit

$$
\lim _{N \rightarrow \infty} \Phi_{11}^{-1}=\frac{2}{\omega^{2}} \int_{0}^{1} \mathrm{~d} x \frac{\sin ^{2}(\pi x)}{v^{2}+4 \sin ^{2}\left(\frac{\pi x}{2}\right)}=\frac{2}{\omega^{2}} \frac{1}{2+v^{2}+\sqrt{v^{2}\left(4+v^{2}\right)}} .
$$

It is then a consequence of (4.15) that

$$
\lim _{N \rightarrow \infty}(N-1) J^{(N)}=\kappa\left(T_{L}-T_{R}\right)
$$

where

$$
\kappa=\frac{\omega^{2}}{\lambda} \frac{1}{2+v^{2}+\sqrt{v^{2}\left(4+v^{2}\right)}} .
$$


Since $J^{(N)}=O\left(N^{-1}\right)$, we get from (4.14) that

$$
\varepsilon_{N}=O\left(N^{-\frac{1}{2}}\right) .
$$

By our discussion in Section 4.2, this implies that in the limit $N \rightarrow \infty$, all the correlation functions involving finitely many terms will converge to the corresponding local equilibrium values if we identify $T_{i}$ with the local temperature of the system at a site $i$.

Summing (4.12) from 1 to $j-1$ and combining it with (4.13) yields

$$
\frac{j-1}{N-1}\left(U_{11}-U_{N N}\right)=U_{11}-U_{j j}+U_{j-1, j+1} .
$$

Then the local equilibrium approximation, Eq. (4.10), shows that

$$
\frac{j-1}{N-1} \Phi_{11}^{-1}\left(T_{L}-T_{R}\right)=T_{L} \Phi_{11}^{-1}-T_{j} \Phi_{j j}^{-1}+T_{j-1} \Phi_{j-1, j+1}^{-1}+O\left(\varepsilon_{N}\right) .
$$

For all $j$ and $k, F_{j-1, k} F_{j+1, k}=F_{j k}^{2}-F_{1 k}^{2}$, and $\Phi^{-1}$ thus satisfies the identity

$$
\Phi_{j-1, j+1}^{-1}=\Phi_{j j}^{-1}-\Phi_{11}^{-1} \text {. }
$$

Since $\left|\Phi_{j j}^{-1}\right| \leqslant 1 / \gamma^{2}$ and $\Phi_{11}^{-1}>0$ uniformly in $N$, we then get the result

$$
T_{j}=T_{L}+\frac{j-1}{N-1}\left(T_{R}-T_{L}\right)+O\left(\varepsilon_{N}\right)
$$

where the correction term vanishes uniformly in $j$ when $N \rightarrow \infty$.

Setting $x=j / N$ the system therefore approaches, in the limit $N \rightarrow \infty$, a local equilibrium state with a temperature profile

$$
T(x)=T_{L}+x\left(T_{R}-T_{L}\right), \quad x \in[0,1] .
$$

Thus Fourier's law holds for the steady state of the system, and the thermal conductivity is given by the temperature independent constant $\kappa$ in (4.18). Note that $\kappa$ remains finite when $v \rightarrow 0$, which points towards a finite conductivity even for the system without the on-site binding potential.

Let us finally remark that we do not think the above bound for $\varepsilon_{N}$ is optimal. Preliminary numerical simulations suggest that $\varepsilon_{N}=O\left(N^{-1}\right)$ rather than $O\left(N^{-\frac{1}{2}}\right)$.

\section{THE GREEN-KUBO FORMULA}

The Green-Kubo formula expresses the local equilibrium conductivity at a position $\mathbf{x}$ with temperature $T(\mathbf{x})$ as an integral over the 
current-current correlations in a (closed) equilibrium system at uniform temperature $T=T(\mathbf{x})$. This corresponds, for the type of stationary state we consider, to a formula for $\kappa$ when $T_{L}$ and $T_{R} \rightarrow T$. Here we verify this formula by an explicit computation. ${ }^{5}$

The following theorem summarizes the results, and shows that the form of the Green-Kubo formula, as defined, e.g., in ref. 2, leads to the correct conductivity for the system with the non-zero on-site potential.

\section{Theorem 5.1. Given $T>0$,}

$\kappa_{\mathrm{GK}}(T)=\frac{1}{T^{2}} \int_{0}^{\infty} \mathrm{d} t \lim _{N \rightarrow \infty} C_{J J}^{(N)}(t ; T)=\lim _{N \rightarrow \infty} \frac{1}{T^{2}} \int_{0}^{\infty} \mathrm{d} t C_{J J}^{(N)}(t ; T)=\kappa$

In the theorem, $\kappa$ is defined by (4.18), and

$$
C_{J J}^{(N)}(t ; T)=\frac{1}{N+1}\langle J(\mathbf{q}(t), \mathbf{p}(t)) J(\mathbf{q}(0), \mathbf{p}(0))\rangle_{S_{T}}
$$

where

$$
J(\mathbf{q}, \mathbf{p})=\sum_{i=1}^{N-1} J_{i}=\sum_{i=1}^{N-1} \frac{\omega^{2}}{2}\left(q_{i}-q_{i+1}\right)\left(p_{i}+p_{i+1}\right) .
$$

The expectation value in (5.2) refers to the stochastic time evolution defined in Section 2 when the initial values $(\mathbf{q}(0), \mathbf{p}(0))$ are distributed according to the equilibrium Gibbs measure at temperature $T$. The proof is a relatively tedious explicit computation, which we do not report here in full detail.

Proof. Define first the matrix $K$ by

$$
(K \mathbf{q})_{i}= \begin{cases}q_{1}-q_{2}, & \text { for } i=1 \\ q_{N-1}-q_{N}, & \text { for } i=N \\ q_{i-1}-q_{i+1}, & \text { otherwise }\end{cases}
$$

${ }^{5}$ It would be possible to derive the Green-Kubo formula also for the present system by using the recent methods developed, e.g., in ref. 9, that is, via a Gallavotti-Cohen type fluctuation theorem. ${ }^{(10)}$ The proof would be a generalization of the one outlined in Section 4 of ref. 11. Our proof however, in addition to being more direct, also gives us a better control on the dependence on the size $N$ of the system. 
so that

$$
\frac{2}{\omega^{2}} J(\mathbf{q}, \mathbf{p})=\sum_{i=1}^{N-1}\left(q_{i}-q_{i+1}\right)\left(p_{i}+p_{i+1}\right)=\mathbf{p}^{T} K \mathbf{q} .
$$

Then $\frac{1}{T^{2}} C_{J J}^{(N)}(t ; T)=\frac{\omega^{4}}{4} g_{N}(t)$ for

$$
g_{N}(t)=\frac{1}{T^{2}(N+1)}\left\langle\mathbf{p}(t)^{T} K \mathbf{q}(t) \mathbf{p}(0)^{T} K \mathbf{q}(0)\right\rangle_{S_{T}} .
$$

The initial equilibrium measure is Gaussian with zero mean and with a covariance $C(0,0)=T E$, where $E=T^{-1} S^{(\mathrm{eq}, T)}$ is by (2.21) independent of $T$. Correspondingly,

$$
C(t, 0)= \begin{cases}T \mathrm{e}^{-t A} E, & \text { when } t \geqslant 0 \\ T E \mathrm{e}^{t A^{T}}, & \text { when } t<0 .\end{cases}
$$

Applying the "pairing rule" of Gaussian correlations and setting

$$
\mathscr{K}=\left(\begin{array}{cc}
0 & K^{T} \\
K & 0
\end{array}\right),
$$

we then obtain, for $t \geqslant 0$,

$$
g_{N}(t)=\frac{1}{2(N+1)} \operatorname{Tr}\left[\mathscr{K} \mathrm{e}^{-t A} E \mathscr{K} E \mathrm{e}^{-t A^{T}}\right],
$$

and, for $t<0, g_{N}(t)=g_{N}(|t|)$.

Let us proceed by assuming the existence of $\lim _{N \rightarrow \infty} g_{N}(t)$ and later comment on how to prove this. From (5.4) we get

$$
\left|g_{N}(t)\right| \leqslant \frac{\operatorname{Tr} I}{2(N+1)}\left\|\mathrm{e}^{-t A}\right\|^{2}\|\mathscr{K}\|^{2}\|E\|^{2} .
$$

Since $\left\|\Phi^{-1}\right\|=\sup _{k} 1 / \mu_{k} \leqslant 1 / \gamma^{2}$, the norm of $E$ is bounded uniformly in $N \rightarrow \infty$. The same is clearly true for $\|\mathscr{K}\|$, and by (2.10) and (5.5) we can now apply dominated convergence in (5.1). This proves the integrability of the limit function, and yields

$$
\kappa_{\mathrm{GK}}=\frac{\omega^{4}}{8} \lim _{N \rightarrow \infty} \frac{1}{N+1} \operatorname{Tr}\left[\mathscr{K} \int_{0}^{\infty} \mathrm{d} t \mathrm{e}^{-t A} E \mathscr{K} E \mathrm{e}^{-t A^{T}}\right] .
$$

We have now proved the first two equalities of the theorem. We note that the above argument, which allows to take the thermodynamic limit 
out of the time-integral, would fail if $\gamma=0$, as then neither the bound on $\|E\|$ nor the exponential decay of $\left\|\mathrm{e}^{-t A}\right\|$ would be uniform in $N$.

Let us then denote

$$
S^{\prime}=\int_{0}^{\infty} \mathrm{d} t \mathrm{e}^{-t A} E \mathscr{K} E \mathrm{e}^{-t A^{T}}=\left(\begin{array}{cc}
U^{\prime} & Z^{\prime} \\
\left(Z^{\prime}\right)^{T} & V^{\prime}
\end{array}\right)
$$

which is possible, as the integral clearly yields a symmetric operator. Then

$$
\operatorname{Tr}\left[\mathscr{K} S^{\prime}\right]=\operatorname{Tr}\left[K^{T}\left(Z^{\prime}\right)^{T}+K Z^{\prime}\right]=2 \operatorname{Tr}\left[\tilde{K} \tilde{Z}^{\prime}\right]
$$

where $\tilde{K}=F^{T} K F$ and $\tilde{Z}^{\prime}=F^{T} Z^{\prime} F$. Like the matrix $S$ defined by (2.11), $S^{\prime}$ is the unique solution of the equation

$$
A S^{\prime}+S^{\prime} A^{T}=E \mathscr{K} E=\left(\begin{array}{cc}
0 & \Phi^{-1} K^{T} \\
\left(\Phi^{-1} K^{T}\right)^{T} & 0
\end{array}\right) .
$$

In Appendix B we prove that

$$
\tilde{Z}_{k l}^{\prime}=-\frac{\lambda}{\omega^{4}} \frac{1}{G\left(c_{k}, c_{l}\right)}\left(\tilde{K}_{-}\right)_{k l},
$$

where $\tilde{K}_{-}$is the antisymmetric part of $\tilde{K}$, and $G$ and $c_{k}$ were defined in Section 2 . In particular, $\tilde{Z}^{\prime}$ is antisymmetric, and thus by (5.7),

$$
\operatorname{Tr}\left[\mathscr{K} S^{\prime}\right]=2 \operatorname{Tr}\left[\tilde{K}_{-} \tilde{Z}^{\prime}\right]=\frac{2 \lambda}{\omega^{4}} \sum_{k, l=1}^{N} \frac{1}{G\left(c_{k}, c_{l}\right)}\left(\tilde{K}_{-}\right)_{k l}^{2} \text {. }
$$

Applying the definitions of $F$ and $K$ and neglecting all symmetric terms, we get after some algebra

$$
\left(\tilde{K}_{-}\right)_{k l}=-\frac{2}{N+1} \delta_{k-l, \text { odd }} \frac{\sin \left(\frac{\pi k}{N+1}\right) \sin \left(\frac{\pi l}{N+1}\right)}{\sin \left(\frac{\pi(k-l)}{2(N+1)}\right) \sin \left(\frac{\pi(k+l)}{2(N+1)}\right)}
$$

where $\delta_{u \text {, odd }}=1$, if is $u$ is odd, and zero, if $u$ is even. Observe then that for $l \approx k$ we have

$$
\left(\tilde{K}_{-}\right)_{k l} \approx-\frac{4}{\pi(k-l)} \delta_{k-l, \text { odd }} \sin \left(\frac{\pi k}{N+1}\right),
$$

while elsewhere $\left(\tilde{K}_{-}\right)_{k l}=O\left(N^{-1}\right)$. Using this observation and the equality $\sum_{u \in \mathbb{Z}} \delta_{u \text {, odd }} / u^{2}=\pi^{2} / 4$, it is possible to prove that

$$
\frac{1}{N+1} \operatorname{Tr}\left[\mathscr{K} S^{\prime}\right]=\frac{8 \lambda}{\omega^{4}} \frac{1}{N+1} \sum_{k=1}^{N} \frac{\omega^{2}}{\lambda^{2}} \frac{\sin ^{2}\left(\frac{\pi k}{N+1}\right)}{v^{2}+4 \sin ^{2}\left(\frac{\pi k}{2(N+1)}\right)}+O\left(\frac{1}{N}\right) .
$$


The same methods can be employed to show that $\lim _{N \rightarrow \infty} g_{N}(t)$ exists for all $t>0$. First write the trace in Eq. (5.4) in the eigenspace of the forcematrix $\Phi$, and then apply the above approximation to $\tilde{K}_{k l}$ to find that only terms with $k \approx l$ contribute and the contribution has a finite limit.

Combining the above with Eq. (5.6), we have now proven that

$$
\kappa_{\mathrm{GK}}=\frac{\omega^{2}}{\lambda} \int_{0}^{1} \mathrm{~d} x \frac{\sin ^{2}(\pi x)}{v^{2}+4 \sin ^{2}\left(\frac{\pi x}{2}\right)}
$$

which shows that $\kappa_{\mathrm{GK}}=\kappa$ for all $T$.

\section{NON-UNIFORM HEAT BATH COUPLING}

Let us now consider the case when the heat bath couplings $\lambda_{i}$ are not all equal and define $\Lambda_{i j}=\delta_{i j} \lambda_{i}$. As long as $\left\|\mathrm{e}^{-t A}\right\|^{2}$ remains integrable, we can repeat the computations in Section 2 and conclude that Eqs. (2.13) for the stationary covariance matrix are still valid. In particular, the matrix $Z$ is then antisymmetric. Therefore, by redoing the computations in Section 2.1, we get the average of the energy transfer $R_{i}$ and of the current $J_{i}$ in the steady state from the equations

$$
\left\langle J_{i}\right\rangle_{S}=\omega^{2} Z_{i, i+1} \quad \text { and } \quad\left\langle R_{i}\right\rangle_{S}=\lambda_{i}\left(T_{i}-V_{i i}\right) .
$$

Thus the self-consistency condition still has the same form as before but, as the earlier explicit solution of the steady state covariance $S$ is no longer possible, redoing the existence, uniqueness and local thermal equilibrium results is not straightforward. On physical grounds, we expect the results to remain valid whenever there is an $N$-independent $\lambda>0$, such that the number of $i$ 's for which $\lambda_{i} \geqslant \lambda$ is proportional to $N$, certainly whenever this is true for all $i$. Instead of trying to redo the proofs, we shall check what happens if we assume that these results hold also when the $\lambda_{i}$ are not all equal.

The equations for the stationary covariance (2.13) now yield the relations

$$
\begin{aligned}
2\left\langle R_{i}\right\rangle_{S} & =2 \omega^{2}\left(Z_{i-1, i}-Z_{i, i+1}\right), \\
\left(\lambda_{i}+\lambda_{i+1}\right) Z_{i, i+1} & =\omega^{2}\left(U_{i i}+U_{i, i+2}-U_{i-1, i+1}-U_{i+1, i+1}\right) .
\end{aligned}
$$

The first equation implies that the current in the self-consistent steady state is constant, and then, by summing (6.2) over $i=1, \ldots, N-1$, we get

$$
\frac{2(N-1) \bar{\lambda}^{(N)}}{\omega^{4}} J^{(N)}=U_{11}-U_{N N}
$$


where

$$
\bar{\lambda}^{(N)}=\frac{1}{N-1}\left(\sum_{i=1}^{N} \lambda_{i}-\frac{\lambda_{N}+\lambda_{1}}{2}\right) .
$$

Let us next assume that the local equilibrium result proved in Section 4.2 is still valid, i.e., that for every $i, j$

$$
U_{i j}=T_{i} \Phi_{i j}^{-1}+O\left(\varepsilon_{N}\right),
$$

where $\varepsilon_{N}$ is defined by Eq. (4.6), and that $\varepsilon_{N} \rightarrow 0$ when $N \rightarrow \infty$. Choosing the $\lambda_{i}$ such that $\lim _{N \rightarrow \infty} \bar{\lambda}^{(N)}=\bar{\lambda}>0$, we get from (6.3) the scaling of the total current,

$$
\lim _{N \rightarrow \infty}(N-1) J^{(N)}=\bar{\kappa}\left(T_{L}-T_{R}\right)
$$

where $\bar{\kappa}$ is given by (4.18), with $\bar{\lambda}$ replacing $\lambda$ in the equation.

The conductivity will in general be space-dependent for non-uniform couplings. Consider, for instance, any sequence of couplings $\lambda_{i}^{(N)} \geqslant 0$ which is bounded (i.e., $\sup _{i, N} \lambda_{i}^{(N)}<\infty$ ) and for which the limit

$$
\Lambda(x)=\lim _{N \rightarrow \infty} \frac{1}{N} \sum_{1 \leqslant i \leqslant N x} \lambda_{i}^{(N)}
$$

exists for all $x \in[0,1]$ and defines a smooth function $\Lambda$ with $\Lambda(1)>0$.

By summing (6.2) over a range of indices from 1 to $j-1$ we get, after applying the local equilibrium assumption and (4.22), that

$$
\frac{2 J^{(N)}}{\omega^{4} \Phi_{11}^{-1}}\left(\sum_{i=1}^{j} \lambda_{i}^{(N)}-\frac{\lambda_{j}^{(N)}+\lambda_{1}^{(N)}}{2}\right)=T_{L}-T_{j}+O\left(\varepsilon_{N}\right) .
$$

Then, by applying (6.3) and (6.6), we can conclude that the temperature profile now converges to

$$
T(x)=T_{L}-\left(T_{L}-T_{R}\right) \frac{\Lambda(x)}{\Lambda(1)},
$$

and, therefore, that Fourier's law is satisfied with a thermal conductivity

$$
\kappa(x)=\frac{\omega^{2}}{\lambda(x)} \frac{1}{2+v^{2}+\sqrt{v^{2}\left(4+v^{2}\right)}}
$$

where $\lambda(x)=\frac{\mathrm{d}}{\mathrm{d} x} \Lambda(x)$. Note that on any interval on which $\lambda(x)=0$ the local conductivity is infinite and the temperature profile remains constant.

For instance, if $\lambda_{i}=\lambda$ for every $m$ :th coupling and $\lambda_{i}=0$ otherwise, we get a finite conductivity equal to $m$ times the one computed in Section 4 . 
Taking $m=N$ we then (formally) recover the linear divergence of the conductivity in $N$ which was found in ref. 5.

\section{HIGHER DIMENSIONS}

Here we extend the results of the previous sections to a system of oscillators first in $d=2$ and then also in higher dimensions. The solution can be obtained in a way very similar to what we did in Sections 2 to 5, and we shall just report the necessary adjustments. Moreover, we shall only consider explicitly the system in two dimensions. No real modifications are necessary to extend the computations to higher dimensions.

The Hamiltonian for the system is now given by

$$
\begin{aligned}
H(\mathbf{q}, \mathbf{p})= & \sum_{i=1}^{N} \sum_{j=1}^{N^{\prime}}\left[\frac{1}{2} p_{i, j}^{2}+u\left(q_{i, j}\right)\right] \\
& +\sum_{j=1}^{N^{\prime}} \sum_{i=1}^{N+1} v\left(q_{i, j}-q_{i-1, j}\right)+\sum_{i=1}^{N} \sum_{j=1}^{N^{\prime}} v\left(q_{i, j}-q_{i, j-1}\right)
\end{aligned}
$$

where we assume, as before, that $q_{0, j}=q_{N+1, j}=0$ and we fix periodic boundary conditions in the second direction, i.e., $q_{i, 0}=q_{i, N^{\prime}}$. As in Section 2 , the oscillator at the site $(i, j)$ is coupled to a Langevin heat bath with temperature $T_{i, j}$, and we set $T_{1, j}=T_{L}, T_{N, j}=T_{R}$ while all other $T_{i, j}$ are to be determined self-consistently. Thus the time evolution is still defined by Eqs. (2.3)-(2.5) if we interpret the operator $\Delta$ in (2.5) as the discrete Laplacian in two dimensions with mixed boundary conditions: Dirichlet in the first direction and periodic in the second direction.

Extending the results in Section 2.1, we first define the local energy by

$$
\begin{aligned}
H_{i, j}(\mathbf{q}, \mathbf{p})= & \frac{1}{2} p_{i, j}^{2}+u\left(q_{i, j}\right)+\frac{1}{2}\left[v\left(q_{i, j}-q_{i-1, j}\right)+v\left(q_{i+1, j}-q_{i, j}\right)\right. \\
& \left.+v\left(q_{i, j}-q_{i, j-1}\right)+v\left(q_{i, j}-q_{i, j+1}\right)\right]
\end{aligned}
$$

again with double contribution for the terms involving $q_{0, j}$ and $q_{N+1, j}$. Then the analog of (2.22) is true if we define the current as a two dimensional vector with $J_{i, j}^{1}=0$ for $i=0, N$, and with the other components given by

$$
\begin{aligned}
& J_{i, j}^{1}(\mathbf{q}, \mathbf{p})=-\frac{\omega^{2}}{2}\left(q_{i+1, j}-q_{i, j}\right)\left(p_{i, j}+p_{i+1, j}\right), \\
& J_{i, j}^{2}(\mathbf{q}, \mathbf{p})=-\frac{\omega^{2}}{2}\left(q_{i, j+1}-q_{i, j}\right)\left(p_{i, j}+p_{i, j+1}\right),
\end{aligned}
$$

where $q_{i, j}$ and $p_{i, j}$ are periodic in $j$. 
The source terms are given by $R_{i, j}=\lambda\left(T_{i, j}-\left(p_{i, j}\right)^{2}\right)$, and the self-consistency condition thus becomes

$$
T_{i, j}=\left\langle\left(p_{i, j}\right)^{2}\right\rangle_{S}, \quad \text { for } \quad i=2, \ldots, N-1 \quad \text { and } \quad j=1, \ldots, N^{\prime}
$$

with $T_{1, j}=T_{L}$ and $T_{N, j}=T_{R}$. The main observation is that, as in ref. 6, we can Fourier transform this system in the periodic direction and obtain a system of decoupled chains.

More precisely, let for $k=1, \ldots, N^{\prime}$

$$
q_{i}(k)=\frac{1}{\sqrt{N^{\prime}}} \sum_{j=1}^{N^{\prime}} q_{i, j} \mathrm{e}^{\mathrm{i} \frac{2 \pi}{N^{\prime}} k j} \quad \text { when } \quad q_{i, j}=\frac{1}{\sqrt{N^{\prime}}} \sum_{k=1}^{N^{\prime}} q_{i}(k) \mathrm{e}^{-\mathrm{i} \frac{2 \pi}{N^{\prime}} k j},
$$

and define $p_{i}(k)$ analogously. This corresponds to a change to a (complex) eigenbasis of the periodic Laplacian, and we obtain that, for any fixed $k$, $\mathbf{q}(k)$ and $\mathbf{p}(k)$ satisfy Eq. (2.3) with the only difference that now the potential $\Phi$ is given by (2.5) with $v^{2}$ replaced by $v(k)^{2}=v^{2}+2\left(1-\cos \left(\frac{2 \pi k}{N^{\prime}}\right)\right) \geqslant v^{2}$. However, the noise term will then become more complicated and it can still a priori couple the components with different values of $k$.

In general, $\mathbf{p}(k)$ and $\mathbf{q}(k)$ are complex numbers, and the stochastic equations should be understood applying to the real and imaginary part separately. However, as $A$ remains a real matrix, Eq. (2.9) still holds if we replace the matrix $\Sigma^{2}$ by

$$
\left(\begin{array}{cc}
0 & 0 \\
0 & \sigma \sigma^{\dagger}
\end{array}\right), \quad \text { where } \quad\left(\sigma \sigma^{\dagger}\right)_{i, k ; i^{\prime}, k^{\prime}}=\delta_{i i^{\prime}} 2 \lambda \frac{1}{N^{\prime}} \sum_{j} T_{i, j} \mathrm{e}^{\mathrm{i} \frac{2 \pi}{N^{\prime}} j\left(k-k^{\prime}\right)}
$$

On the other hand, our bound for the norm of the exponential of $A$ is obviously still valid, and we can conclude that for every temperature profile there is a unique stationary state which is reached exponentially fast and which is determined by Eq. (2.11) with the matrix (7.6) replacing $\Sigma^{2}$ there.

Next we need to prove the existence and uniqueness of the self-consistent temperature profile. In fact, Theorem 3.1 is valid also in the higher dimensional case considered here, but since the proof remains essentially unchanged, we do not include it here.

The boundary conditions we impose are constant in the periodic direction, and we expect from symmetry that the self-consistent temperature profile is also constant in that direction, even for finite $N$. This is also directly implied by the above quoted uniqueness since, if $\left(t_{i, j}\right)$ is a self-consistent profile, then also its translates, $T_{i, j}=t_{i, j+j^{\prime}}$ for any $j^{\prime}$, are self-consistent with the same boundary conditions. 
Consider thus a temperature profile $T_{i, j}=\tau_{i}$ for which $\tau_{1}=T_{L}$ and $\tau_{N}=T_{R}$. By (7.6), we then have always

$$
\left(\sigma \sigma^{\dagger}\right)_{i, k ; i^{\prime}, k^{\prime}}=\delta_{i i^{\prime}} \delta_{k k^{\prime}} 2 \lambda \tau_{i}
$$

Applying this in the equation corresponding to (2.12) reveals that the components having different values of $k$ then become independent in the steady state. In particular,

$$
\left\langle p_{i}(k) p_{j}\left(k^{\prime}\right)^{*}\right\rangle_{S}=\left\langle p_{i}(k) p_{j}(k)^{*}\right\rangle_{S} \delta_{k k^{\prime}},
$$

and, therefore for all $i, j$,

$$
\left\langle p_{i, j} p_{i, j}\right\rangle_{S}=\frac{1}{N^{\prime}} \sum_{k, k^{\prime}=1}^{N^{\prime}} \mathrm{e}^{-\mathrm{i} \frac{2 \pi}{N^{\prime}} j\left(k-k^{\prime}\right)}\left\langle p_{i}(k) p_{i}\left(k^{\prime}\right)^{*}\right\rangle_{S}=\frac{1}{N^{\prime}} \sum_{k=1}^{N^{\prime}}\left\langle p_{i}(k) p_{i}(k)^{*}\right\rangle_{S} .
$$

Here the expectation value can be computed by $\left\langle p_{i}(k) p_{i}(k)^{*}\right\rangle_{S}=(M(k) \tau)_{i}$, where $M(k)=\left.M\right|_{v^{2}=v(k)^{2}}$ and $M$ is the matrix defined in Section 3 . Therefore, simply by replacing the matrix $M$ with $\left(N^{\prime}\right)^{-1} \sum_{k} M(k)$ we can repeat the computations in Section 3, and find a vector $\tau$ which leads to a self-consistent profile $T_{i, j}$.

It is then easy to see, applying the decoupling of the modes as above and then using the antisymmetry of the covariance component $Z$, that there is no average current in the second direction, i.e., $\left\langle J_{i, j}^{2}\right\rangle_{S}=0$. Similarly, we get for all $i=1, \ldots, N-1$ the result

$$
\left\langle J_{i, j}^{1}\right\rangle_{S}=\omega^{2} Z_{i, j ; i+1, j}=\left.\frac{1}{N^{\prime}} \sum_{k=1}^{N^{\prime}} J^{(N)}\right|_{v^{2}=v(k)^{2}}
$$

where $J^{(N)}$ denotes the current through the corresponding chain.

Repeating the computations in Section 4 and using the above decoupling of the $k$-modes, we can then conclude that local equilibrium holds in the limit $N, N^{\prime} \rightarrow \infty$ (for this one needs to notice that the exponential decay of correlations is uniform in $k$, as $v(k)^{2} \geqslant v^{2}>0$ ), the limiting temperature profile is constant in the periodic direction and connects $T_{L}$ and $T_{R}$ linearly in the first direction. Fourier's law is also satisfied with the conductivity now given by

$$
\kappa=\lim _{N^{\prime} \rightarrow \infty} \frac{1}{N^{\prime}} \sum_{k} \kappa(k)=\frac{\omega^{2}}{\lambda} \int_{0}^{1} \frac{\mathrm{d} y}{2+\tilde{v}(y)^{2}+\sqrt{\tilde{v}(y)^{2}\left(4+\tilde{v}(y)^{2}\right)}}
$$

where $\tilde{v}(y)^{2}=v^{2}+2(1-\cos (2 \pi y))$. 
For the system with $d-1$ extra periodic dimensions, we could analogously arrive at the same conclusions, but with a conductivity

$$
\kappa=\frac{\omega^{2}}{\lambda} \int_{[0,1]^{d-1}} \frac{\mathrm{d}^{d-1} y}{2+\tilde{v}(\mathbf{y})^{2}+\sqrt{\tilde{v}(\mathbf{y})^{2}\left(4+\tilde{v}(\mathbf{y})^{2}\right)}}
$$

where now $\tilde{v}(\mathbf{y})^{2}=v^{2}+2 \sum_{i=1}^{d-1}\left(1-\cos \left(2 \pi y_{i}\right)\right)$. Observe, in particular, that the conductivity steadily decreases with each added dimension. We prove in Appendix $\mathrm{C}$ that the asymptotic behavior of the conductivity in the limit $d \rightarrow \infty$ is given by

$$
\kappa=\frac{\omega^{2}}{4 d \lambda}(1+o(1))
$$

where the correction term depends only on $v$ and $d$. Thus by choosing a suitable sequence of $\lambda=O\left(d^{-1}\right)$, we can have $\lambda \rightarrow 0$ when $d \rightarrow \infty$ and still keep the conductivity finite and constant.

It would also be straightforward to check that the Green-Kubo formula holds in the higher dimensional case. More precisely, Theorem 5.1 is still valid for the above system in $d$ dimensions, if the current-current correlator is defined instead of (5.2) by

$$
C_{J J}^{(N)}(t ; T)=\frac{1}{d \prod_{i} N_{i}}\langle\mathbf{J}(\mathbf{q}(t), \mathbf{p}(t)) \cdot \mathbf{J}(\mathbf{q}(0), \mathbf{p}(0))\rangle_{S_{T}} .
$$

For proving this, the $\left\langle J^{1} J^{1}\right\rangle$-term can be analyzed exactly as before, while the analysis of the remaining $\left\langle J^{i} J^{i}\right\rangle$-terms in the periodic directions will be even simpler, as in the complex eigenbasis used here the operator corresponding to $K$ will be exactly diagonal.

\section{DISCUSSION}

We raise again the question, discussed extensively in ref. 1 and 12, of whether it is possible to derive Fourier's law for a system with purely Hamiltonian bulk dynamics. There are two ways of formulating this problem: (i) The system could be fully isolated and evolving towards equilibrium from an initial nonuniform local equilibrium state. (ii) The system could be maintained in a stationary non-equilibrium state by coupling it at the boundaries to infinite reservoirs, either stochastically as in ref. 5 (or variations thereof, see ref. 1) or mechanically as in refs. 13 and 14. One could also keep the temperature fixed at the end of the system by means of deterministic Gaussian thermostats. ${ }^{(10)}$ 
In the first case this amounts to proving the existence of a hydrodynamical scaling limit on the dissipative time scale. This is a well known, extremely difficult problem. ${ }^{(7)}$ It is clearly not true for the harmonic crystal or other integrable models but is believed to be true for macroscopic systems with more realistic type of interactions, e.g., hard spheres or with Lennard-Jones potentials. For anharmonic crystals, the kind considered in ref. 14, one would have to go beyond the Kolmogorov, Arnold, Moser domain $^{(15)}$ and presumably also beyond the Fermi, Pasta, Ulam ${ }^{(16)}$ models. ${ }^{(2)}$ The only mechanical system, for which such a result has been derived, is for the highly degenerate model of a macroscopic system of independent particles moving in a periodic array of scatterers, i.e., for the multi-particle Sinai billiard, where one proves Fick's law, the analog of Fourier's law for the conserved particle current. ${ }^{(17)}$

In the second case of stationary non-equilibrium states one may hope to prove a global Fourier's law, i.e., we want $\mathscr{L} J_{\mathscr{L}} /\left(T_{L}-T_{R}\right) \rightarrow \kappa$ as $\mathscr{L} \rightarrow \infty$. Here $\mathscr{L}$ is the distance, in microscopic units, between the boundaries of the system, say a cylinder, maintained at fixed temperatures $T_{L}$ and $T_{R}$. We want a $\kappa$ which depends only on the bulk properties of the system. We expect further that when $T_{L} \rightarrow T_{R}=T$, the limit of $\kappa$ should coincide with the heat conductivity $\kappa(T)$ at the local equilibrium temperature $T$ in the isolated time-evolving case (i). Again the only mechanical system for which such a result has been proven is for the degenerate system of point particles moving among a periodic array of scatterers where the heat current is really a particle current (particles pick up energy at the right wall). ${ }^{(13)}$ The best that has been proven for other systems is the existence of a stationary state ${ }^{(14,18,19)}$ and the positivity of $\left(T_{L}-T_{R}\right) J_{\mathscr{L}}$ for fixed $\mathscr{L} .^{(20)}$

The results proven in this paper make use of the stochastic interactions in the bulk to produce a local equilibrium state. This is in the spirit of the general work in the last two decades proving the existence of hydrodynamical laws in the appropriate scaling limits for systems evolving via stochastic dynamics. ${ }^{(7)}$ We should mention here in particular the work of Kipnis, Marchioro, and Presutti ${ }^{(21)}$ who proved results similar to ours for a model with purely stochastic internal dynamics. They were in fact able to consider a situation where the energy is strictly conserved in the bulk rather than just in the average as in the model considered here. This can be done also for a modified (more mechanical) version of their model considered by $\mathrm{Olla}^{(22)}$ in which there is an energy conserving Ornstein-Uhlenbeck type process producing an energy exchange between neighboring oscillators.

The main advantage of the BRV self-consistent model is that the average energy flow along the temperature gradient is, as seen in (2.23), entirely Hamiltonian. As mentioned in the introduction, it might in fact be possible to make our model entirely mechanical by coupling each site to a 
large Hamiltonian reservoir, a la Ford, Kac, and Mazur, ${ }^{(23)}$ which would produce an effective stochastic reservoir that would automatically, without any imposition of self-consistency, be at the right temperature.

This is in fact what seems to happen effectively when we let the dimension of the crystal go to infinity. As shown in Appendix C, after taking the limits $t \rightarrow \infty$ and $N \rightarrow \infty$, we can let the coupling to the interior heat baths, which we denote by $\ell_{d}$, go to zero as $d^{-1}$, and still obtain a finite value of the conductivity. It is clear from the analysis in Section 6 that, if we set $\lambda_{1}=\lambda_{N}=\ell_{0}$ and $\lambda_{2}=\lambda_{3}=\cdots=\lambda_{N-1}=\ell_{d}$, then the heat conductivity is obtained by replacing $\lambda$ by $\ell_{d}$ in (4.18) and in (7.10).

An open interesting problem is to consider our model for an anharmonic crystal, e.g., by setting in (2.2), $u(q)=\frac{1}{2} \gamma^{2} q^{2}+\frac{1}{2} \delta q^{4}$. We expect that for a fixed $\delta>0$ the heat conductivity $\kappa$ would have a finite limit as the auxiliary couplings with the interior heat baths are taken to zero. It might even be possible to prove such a result by starting with a perturbation expansion in $\delta$ around the local equilibrium stationary state found here and then doing a suitable resummation or applying a renormalization group type argument. See however, the results of the perturbation expansion in the case with purely Hamiltonian bulk dynamics derived in ref. 24 .

We note finally that the harmonic heat conductivity $\kappa$ given in (4.18) would remain finite if we let $\lambda \rightarrow 0$ and $\gamma \rightarrow \infty$ in such a way that $\lambda \gamma^{2} \rightarrow \alpha>0$. It is not clear whether this limit has any physical significance.

\section{APPENDIX A: BOUND FOR THE TIME-EVOLUTION MATRIX}

Let $F$ be the orthogonal matrix defined by Eq. (2.15), and define

$$
\mathscr{F}=\left(\begin{array}{ll}
F & 0 \\
0 & F
\end{array}\right) .
$$

As $F$ diagonalizes $\Phi$, we then easily see that $A=\mathscr{F} \tilde{A}^{T}$, where (with $Y$ again denoting the eigenvalue matrix of $\Phi$ )

$$
\tilde{A}=\left(\begin{array}{cc}
0 & -I \\
Y & \lambda I
\end{array}\right)
$$

Since $\tilde{A}$ is block diagonal (after a permutation of indices) and $\mathscr{F}$ is orthogonal, it follows that the norm of the exponential satisfies

$$
\left\|\mathrm{e}^{-t A}\right\|=\max _{k}\left\|\mathrm{e}^{-t A_{k}}\right\|
$$


where for each $k$ we have defined

$$
A_{k}=\left(\begin{array}{cc}
0 & -1 \\
\mu_{k} & \lambda
\end{array}\right)
$$

The eigenvalues of $A_{k}$ are

$$
\alpha_{k}^{ \pm}=\frac{\lambda}{2} \pm \rho_{k} \quad \text { where } \quad \rho_{k}=\sqrt{\frac{\lambda^{2}}{4}-\mu_{k}},
$$

and it is easy to see that

$$
\operatorname{Re} \alpha_{k}^{ \pm} \geqslant \underline{\alpha}=\min \left\{\frac{\lambda}{2}, \frac{\gamma^{2}}{\lambda}\right\}>0 .
$$

However, since $A_{k}$ is not symmetric (in fact, there are values of the parameters when it is not even diagonalizable) we have to take more care in analyzing the norm of its exponential. Performing the Jordan decomposition of $A_{k}$ explicitly yields

$$
\mathrm{e}^{-t A_{k}}=\mathrm{e}^{-t \lambda / 2} \cosh \left(\rho_{k} t\right)\left[I+\frac{\tanh \left(\rho_{k} t\right)}{\rho_{k}}\left(\begin{array}{cc}
\lambda / 2 & 1 \\
-\mu_{k} & -\lambda / 2
\end{array}\right)\right]
$$

from which we straightforwardly arrive at the following bound valid for $t \geqslant 0$,

$$
\left\|\mathrm{e}^{-t A_{k}}\right\| \leqslant \mathrm{e}^{-t \alpha}\left[1+t\left(1+\gamma^{2}+4 \omega^{2}+\lambda / 2\right)\right] .
$$

Applying this to (A.2) easily yields the conclusion in Section 2, at Eq. (2.10). We remark that if $\gamma=0$, we could still have a lower bound $\underline{\alpha}>0$, but it would not be uniform in $N$. In fact, since then $\inf _{k} \mu_{k}=O\left(N^{-2}\right)$, we would then need to take also $\underline{\alpha}=O\left(N^{-2}\right)$.

\section{APPENDIX B: SOLUTION OF THE STATIONARY COVARIANCE}

We derive here an explicit solution to the equation

$$
A S+S A^{T}=\Sigma^{2},
$$

which-for the matrix $\Sigma^{2}$ used in Section 2-will yield the stationary covariance matrix. The matrix $A$ is defined as in (2.4), but we need the 
solution for a more general "noise matrix" in Section 5. Therefore, we consider here

$$
\Sigma^{2}=\left(\begin{array}{cc}
0 & b \\
b^{T} & 2 \lambda d
\end{array}\right)
$$

where $b$ and $d$ are real $N \times N$ matrices and $d^{T}=d$.

Denoting

$$
S=\left(\begin{array}{ll}
U & Z \\
Z^{T} & V
\end{array}\right),
$$

we get that $S$ is a solution to (B.1) if and only if its components satisfy

$$
\begin{aligned}
Z^{T} & =-Z \\
V & =\frac{1}{2}(\Phi U+U \Phi)-b_{+} \\
\lambda Z & =\frac{1}{2}(\Phi U-U \Phi)+b_{-} \\
2 \lambda\left(d+b_{+}\right) & =\Phi Z-Z \Phi+\lambda(U \Phi+\Phi U)
\end{aligned}
$$

where $b_{+}$and $b_{-}$are the symmetric and the antisymmetric part of $b$.

If we define

$$
\tilde{d}=F^{T} d F \quad \text { and } \quad \tilde{V}=F^{T} V F,
$$

and also $\tilde{b}, \tilde{U}$, and $\tilde{Z}$ similarly, then we get

$$
\begin{aligned}
& \tilde{U}_{k l}=\frac{2}{g_{k l}}\left[2 \lambda^{2}\left(\tilde{d}_{k l}+\left(\tilde{b}_{+}\right)_{k l}\right)-\left(\mu_{k}-\mu_{l}\right)\left(\tilde{b}_{-}\right)_{k l}\right] \\
& \tilde{V}_{k l}=\frac{1}{g_{k l}}\left[2 \lambda^{2}\left(\mu_{k}+\mu_{l}\right) \tilde{d}_{k l}-\left(\mu_{k}-\mu_{l}\right)\left(\mu_{k} \tilde{b}_{k l}-\mu_{l} \tilde{b}_{l k}\right)\right] \\
& \tilde{Z}_{k l}=\frac{2 \lambda}{g_{k l}}\left[\left(\mu_{k}-\mu_{l}\right) \tilde{d}_{k l}+\mu_{k} \tilde{b}_{k l}-\mu_{l} \tilde{b}_{l k}\right]
\end{aligned}
$$

where $\mu_{k}$ are the eigenvalues of $\Phi$, and for all $k$ and $l$

$$
g_{k l}=4 \omega^{4} G\left(c_{k}, c_{l}\right)>0
$$

where $c_{k}$ and $G$ are defined in Eqs. (2.18) and (2.20), respectively. 
When $b=0$ and $d_{i j}=\delta_{i j} T_{i}$ we get (2.16)-(2.19). In Section 5 we need to know $\tilde{Z}_{k l}$ when $d=0$ and $b=\Phi^{-1} K^{T}$ with $K$ defined by (5.3). Since then $\tilde{b}_{k l}=\tilde{K}_{l k} / \mu_{k}$, where $\tilde{K}=F^{T} K F$, we get

$$
\tilde{Z}_{k l}=-\frac{\lambda}{\omega^{4}} \frac{1}{G\left(c_{k}, c_{l}\right)}\left(\tilde{K}_{-}\right)_{k l}
$$

with $\tilde{K}_{-}$denoting the antisymmetric part of $\tilde{K}$.

\section{APPENDIX C: ASYMPTOTIC BEHAVIOR OF THE CONDUCTIVITY}

In Section 7 we derived a formula for the conductivity of the $d$-dimensional crystal,

$$
\kappa=\frac{\omega^{2}}{\lambda} I \quad \text { where } \quad I=\int_{[0,1]^{d-1}} \frac{\mathrm{d}^{d-1} y}{2+\tilde{v}(\mathbf{y})^{2}+\sqrt{\tilde{v}(\mathbf{y})^{2}\left(4+\tilde{v}(\mathbf{y})^{2}\right)}}
$$

and $\tilde{v}(\mathbf{y})^{2}=v^{2}+2 \sum_{i=1}^{d-1}\left(1-\cos \left(2 \pi y_{i}\right)\right)$. Here we prove that the asymptotic behavior of $I$ for $d \rightarrow \infty$ is given for any fixed $v>0$ by

$$
I=\frac{1}{4 d}(1+o(1))
$$

First we point out that for all $r \geqslant 0$

$$
\frac{1}{2+r^{2}+\sqrt{r^{2}\left(4+r^{2}\right)}}=\int_{0}^{1} \mathrm{~d} x \frac{\sin ^{2}(\pi x)}{r^{2}+4 \sin ^{2}\left(\frac{\pi x}{2}\right)}=\int_{0}^{1} \mathrm{~d} x \frac{\sin ^{2}(2 \pi x)}{r^{2}+4 \sin ^{2}(\pi x)}
$$

and, therefore,

$$
I=\int_{[0,1]^{d}} \mathrm{~d}^{d} y \frac{\sin ^{2}\left(2 \pi y_{1}\right)}{v^{2}+4 \sum_{i=1}^{d} \sin ^{2}\left(\pi y_{i}\right)} .
$$

Since the denominator is always strictly positive, we can then use the formula $1 / r=\int_{0}^{\infty} \mathrm{d} t \exp (-t r)$ valid for all $r>0$ and obtain

$$
I=\int_{0}^{\infty} \mathrm{d} t \mathrm{e}^{-t v^{2}} I_{1}(t) I_{0}(t)^{d-1}
$$

where

$$
\begin{aligned}
& I_{0}(t)=\int_{0}^{1} \mathrm{~d} y \mathrm{e}^{-4 t \sin ^{2}(\pi y)}, \quad \text { and } \\
& I_{1}(t)=\int_{0}^{1} \mathrm{~d} y \sin ^{2}(2 \pi y) \mathrm{e}^{-4 t \sin ^{2}(\pi y)} .
\end{aligned}
$$


Now both functions $I_{i}(t), i=0,1$, are clearly continuous and strictly monotonously decreasing from $I_{i}(0)$ to 0 when $t$ goes from 0 to $\infty$, with $I_{0}(0)=1$ and $I_{1}(0)=\frac{1}{2}$. In addition, $I_{0}$ is bounded for all $t \geqslant 0$ by

$$
I_{0}(t) \leqslant \frac{1}{\sqrt{1+t}} .
$$

This follows from

$$
\begin{aligned}
I_{0}(t) & =\int_{0}^{1} \mathrm{~d} x \mathrm{e}^{-4 t \sin ^{2}(\pi x / 2)} \\
& \leqslant \int_{0}^{1} \mathrm{~d} x \mathrm{e}^{-4 t x^{2}}=\frac{1}{\sqrt{1+t}} \int_{0}^{\sqrt{1+t}} \mathrm{~d} y \mathrm{e}^{-4 y^{2} t /(1+t)}
\end{aligned}
$$

since the derivative of the last integral is negative for $t \geqslant 0$.

By dominated convergence we then find that, when $d \rightarrow \infty$,

$$
d \int_{1}^{\infty} \mathrm{d} t \mathrm{e}^{-t v^{2}} I_{1}(t) I_{0}(t)^{d-1} \rightarrow 0
$$

Changing variables to $s=t d$ in the remaining integral shows then that

$$
d I=\int_{0}^{d} \mathrm{~d} s \mathrm{e}^{-v^{2} s / d} I_{1}\left(\frac{s}{d}\right) I_{0}\left(\frac{s}{d}\right)^{d-1}+o(1) .
$$

Since $1+x \geqslant 2^{x}$ for all $0 \leqslant x \leqslant 1$, inequality (C.2) yields the bound

$$
I_{0}\left(\frac{s}{d}\right)^{d-1} \leqslant 2^{-s / 4}
$$

for all $0 \leqslant s \leqslant d$ and $d \geqslant 2$. This means that dominated convergence can also be applied to the integral in (C.3), and as $I_{0}(s / d)=1-2 s / d+O\left(d^{-2}\right)$, we then find

$$
\lim _{d \rightarrow \infty}(d I)=\frac{1}{2} \int_{0}^{\infty} \mathrm{d} s \mathrm{e}^{-2 s}=\frac{1}{4}
$$

which proves the Eq. (C.1).

\section{ACKNOWLEDGMENTS}

We would like to thank the Institute for Advanced Study in Princeton, New Jersey, USA, for generous hospitality making this project possible. 
We also want to thank Antti Kupiainen, Luc Rey-Bellet, and Herbert Spohn for helpful discussions. J. Lukkarinen acknowledges the financial support for this project by the Academy of Finland Grants Nr. 100438 and Nr. 200231. This work was also supported by NSF Grant DMR 01-279-26 and by AFOSR Grant 49620-01-1-0154.

\section{REFERENCES}

1. F. Bonetto, J. L. Lebowitz, and L. Rey-Bellet, Fourier's law: A challenge to theorists, in Mathematical Physics 2000, A. Fokas, A. Grigoryan, T. Kibble, and B. Zegarlinski, eds. (Imperial College Press, London, 2000), pp. 128-150.

2. S. Lepri, R. Livi, and A. Politi, Thermal conduction in classical low-dimensional lattices, Phys. Rep. 377:1-80 (2003).

3. M. Bolsterli, M. Rich, and W. M. Visscher, Simulation of nonharmonic interactions in a crystal by self-consistent reservoirs, Phys. Rev. A 4:1086-1088 (1970).

4. M. Rich and W. M. Visscher, Disordered harmonic chain with self-consistent reservoirs, Phys. Rev. B 11:2164-2170 (1975).

5. Z. Rieder, J. L. Lebowitz, and E. Lieb, Properties of a harmonic crystal in a stationary nonequilibrium state, J. Math. Phys. 8:1073-1078 (1967).

6. H. Nakazawa, On the lattice thermal conduction, Suppl. Progr. Theor. Phys. 45:231-262 (1970).

7. H. Spohn, Large Scale Dynamics of Interacting Particles (Springer, Berlin, 1991).

8. B. Øksendal, Stochastic Differential Equations: An Introduction with Applications, 5th Ed. (Springer, Berlin, 1998).

9. J. L. Lebowitz and H. Spohn, A Gallavotti-Cohen type symmetry in the large deviation functional for stochastic dynamics, J. Stat. Phys. 95:333-365 (1999).

10. G. Gallavotti and E. G. D. Cohen, Dynamical Ensembles in Stationary States, J. Stat. Phys. 80:931-970 (1995).

11. L. Rey-Bellet, Statistical mechanics of anharmonic lattices (2003), preprint, http://arxiv.org/abs/math-ph/0303021.

12. J. L. Lebowitz, Exact results in nonequilibrium statistical mechanics: Where do we stand? Suppl. Progr. Theor. Phys. 64:35-49 (1979).

13. J. L. Lebowitz and H. Spohn, Transport properties of the Lorentz gas: Fourier's law, J. Stat. Phys. 19:633-654 (1978).

14. J.-P. Eckmann, C.-A. Pillet, and L. Rey-Bellet, Non-equilibrium statistical mechanics of anharmonic chains coupled to two heat baths at different temperatures, Commun. Math. Phys. 201:657-697 (1999).

15. M. Tabor, Chaos and Integrability in Nonlinear Dynamics: An Introduction (Wiley, New York, 1989).

16. E. Fermi, J. Pasta, and S. Ulam, Studies in nonlinear problems, I, in Nonlinear Wave Motion, A. C. Newell, ed. (American Mathematical Society, Providence, RI, 1974), pp. 143-156. Originally published as Los Alamos Report LA-1940 in 1955.

17. J. L. Lebowitz and H. Spohn, Microscopic basis for Fick's law of self-diffusion, J. Stat. Phys. 28:539-556 (1982).

18. S. Goldstein, J. L. Lebowitz, and E. Presutti, Stationary states for a mechanical system with stochastic boundaries, in Random Fields (Colloquia Mathematicae Societatis János Bolyai 27), J. Fritz, J. L. Lebowitz, and D. Szász, eds. (North-Holland, Amsterdam, 1981), pp. 403-419. 
19. S. Goldstein, C. Kipnis, and N. Ianiro, Stationary states for a system with stochastic boundary conditions, J. Stat. Phys. 41:915-939 (1985).

20. L. Rey-Bellet and L. E. Thomas, Fluctuations of the entropy production in anharmonic chains, Ann. H. Poinc. 3:483-502 (2002).

21. C. Kipnis, C. Marchioro, and E. Presutti, Heat flow in an exactly solvable model, J. Stat. Phys. 27:65-74 (1982).

22. S. Olla, private communication.

23. G. W. Ford, M. Kac, and P. Mazur, Statistical mechanics of assemblies of coupled oscillators, J. Math. Phys. 6:504-515 (1965).

24. R. Lefevere and A. Schenkel, Perturbative analysis of anharmonic chains of oscillators out of equilibrium (2003), preprint, http://arxiv.org/abs/math-ph/0303050. 